\title{
Tissue Mitral Valve
}

National Cancer Institute

\section{Source}

National Cancer Institute. Tissue Mitral Valve. NCI Thesaurus. Code C80471.

A valve made of animal tissues or harvested from human cadavers and used to replace the mitral valve in patients with mitral valve stenosis or regurgitation. 